\title{
Improving presurgical evaluation with regards to emotional cognition
}

Volume 7 Issue 5 - 2017

Jeffrey L Hatcher
Department of psychology, USA

Correspondence: Jeffrey L Hatcher, Department of psychology, 137 Spofford Rd. Boxford, MA 01921, USA, Email jeffreyleehatcher@gmail.com

Received: May 15, 2017 | Published: May 15, 2017 patient due to the chance of cognitive impairment. While doctors focus on assessing lateral dominance for language, not every patient thinks most about linguistic impairment. As a patient, my chief anxiety is for emotional impairment. Our brains are bilaterally redundant in motor function and sensory processing. Unfortunately for surgical candidates, brains do not come with symmetrical redundancy in those networks giving rise to our personalities. There is no firmly elucidated bilateral symmetry in loving your child. Extensive research has been done to understand the differing contributions of both cerebral hemispheres in recognizing the emotional content of faces, voices, and a vast array of sensory stimuli. However, the work with music done by Gosselin et al., ${ }^{1} \&$ Khalfa et al., ${ }^{2}$ on surgically treated patients encapsulates the issues of emotion in a methodologically eloquent manner. Music is a prolonged acoustic stimulus with emotional content. With it, they have demonstrated risks for surgical impairment of emotional recognition abilities. Both studies examined people with temporal lobe resections. Gosselin et al., ${ }^{1}$ played music chosen to elicit feelings of fear, peace, happiness, or sadness to volunteers with right or left temporal resections and also to healthy controls. Subjects then rated each passage with respect to these categories of emotion. In the similar study of Khalfa et al., ${ }^{2}$ music was chosen to inspire happiness, sadness, or anger. Gosselin et al., ${ }^{1}$ report that persons having resections on either side correctly identified happy and sad music but showed ambivalence in recognizing scary or peaceful music. Different resections caused differing levels of errors. For example, some right side resectioned persons mistook scary music for peaceful. The authors found a general impairment in recognizing scary music. Happiness recognition was relatively unaffected by surgery. Change in peaceful and sad music recognition was ambiguous in what erroneous emotion was perceived. Overall, the authors believe that resection can impair a patient's ability to perceive danger. Khalfa et al., ${ }^{2}$ found impairment in right - resected persons' ability to recognize sadness and left - resected persons' ability to recognize happiness and anger. Right - resected individuals' unrecognized sad passages were mistaken as angry. Left - resected individuals misassigned unrecognized happy passages as either sad or angry. However, more than $75 \%$ of assignments were correct for resected patients. The authors suggest that positive music recognition is a left temporal lobe process, whereas negative recognition comes about bilaterally. As a patient, I cannot refrain from extrapolating an impairment to recognizing the emotional state of a loved one by their voice tonality. Emotional recognition that appears to lateralize does not do so in identical ways for all people. ${ }^{1,2}$ I want to have as much personalized information on all forms of risk as I can get. So what to do? I see no prima faciereason for believing that Wada's intracarotid amobarbital test cannot help address these issues. Music has been productively used in published research, and it could be clinically used with similar methodologies. Furthermore, facial

recognition of emotion has been long researched, hence a visual test should also be optimized, perhaps using the Japanese and Caucasian Brief Affect Recognition Test. ${ }^{3}$ Be yond quizzing, a useful metric in such testing might be electrodermal activity used in the manner of Kubota et al., ${ }^{4}$ It reveals psychological arousal and though it does not pinpoint the emotions specifically elevated ${ }^{4}$ it does respond strongly to a specific set of them. ${ }^{5}$ Notably, the useful application of electrodermal monitoring is not dependent upon a conscious ability to follow instructions. An expansion of Wada testing applications might psychologically complicate the decision-making process for some patients. Moderating the stress of the process is a useful function for psychiatrists. Psychiatrists need more inclusion such as things may be aside from just my proposals. ${ }^{67}$ Quickly making the assumption that patients will suffer more stress from having more information is uncalled for. ${ }^{7}$ Presuming that language tops the list of patient concerns about resection is wise. However, after status epilepticus, some of us have experienced temporary emotional detachment from loved ones. This experience can unleash a flood of suicidal ideation, and dead people care little about linguistic lateralization. I would rather continue to hope for better medication options than hasten to surgery and risk compromising emotional cognition. Incorporating psychological research methodologies into the Wada exam could help assess that risk.

\section{Acknowledgments}

None.

\section{Conflicts of interest}

Author declares there are no conflicts of interest.

\section{Funding}

None. 


\section{References}

1. Gosselin N, Isabelle Peretz, Marion Noulhiane, et al. Impaired Recognition of Scary Music Following Unilateral Temporal Lobe Excision. Brain. 2005;128(pt 3):628-640.

2. Khalfa S, Charles Delbe, Emmanuel Bigand, et al. Positive and Negative Music Recognition Reveals a Specialization of Mesio-Temporal Structures in Epileptic Patients. Music Perception. 2008;25(4):295-302.

3. Matsumoto D, Jeff Le Roux, Carinda Wilson Cohn, et al. A New Tes to Measure Emotion Recognition Ability: Matsumoto and Ekman's Japanese and Caucasian Brief Affect Recognition Test (JACBART) Journal of Nonverbal Behavior. 2000;24(3):179-209.
4. Kubota Y, Sato W, Murai T, et al. Emotional Cognition without Awareness after Unilateral Temporal Lobectomy in Humans. JNeurosci. 2000;20(19):RC97.

5. Lanteaume L, Khalfa S, Regis J, et al. Emotion Induction after Direct Intracerebral Stimulations of Human Amygdala. Cerebral Cortex. 2007;17(6):1307-1313.

6. Koch-Stoecker S. Psychiatric Effects of Surgery for Temporal Lobe Epilepsy. In: Trimble et al. (Eds.) The Neuropsychiatry of Epilepsy, Cambridge University Press, Cambridge, UK. 2002. p.266-282.

7. Hatcher JL. Tacking on the Styx: an Epileptic Sails the Facts, Fiction, and Philosophy of a Mental Illness, Author house, Indiana, USA. 2016. p. $1-300$. 